\title{
Computation of Five-Dimensional Grain Boundary Energy
}

\author{
Yong-Kun Luo $\cdot$ Rong-Shan Qin
}

Received: 21 October 2014/Revised: 27 November 2014/Published online: 24 February 2015

(c) The Chinese Society for Metals and Springer-Verlag Berlin Heidelberg 2015

\begin{abstract}
A broken-bond type computational method has been developed for the calculation of the five-dimensional grain boundary energy. The model allows quick quantification of the unrelaxed five-dimensionally specified grain boundary energy in arbitrary orientations. It has been validated on some face-centred cubic metals. The stereo projections of grain boundary energy of $\sum 3, \sum 5, \sum 7, \sum 9, \sum 11, \sum 17 \mathrm{~b}$ and $\sum 31 \mathrm{a}$ have been studied. The results of Ni closely resemble experimentally determined grain boundary energy distribution figures, suggesting that the overall anisotropy of grain boundary energy can be reasonably approximated by the present simple model. Owing to the overlooking of relaxation matter, the absolute values of energy calculated in present model are found to be higher than molecular dynamic-based results by a consistent magnitude, which is $1 \mathrm{~J} / \mathrm{m}^{2}$ for Ni. The coverage of present method forms a bridge between atomistic and meso-scale simulations regarding polycrystalline microstructure.
\end{abstract}

KEY WORDS: Boundaries; Grain boundary energy; Grain boundary structure

\section{Introduction}

Ten years ago, in situ observation of growing single crystals was made possible by a real-time three-dimensional $\mathrm{X}$-ray diffraction technique [1]. Owing to recent advances in materials characterization methods, $3 \mathrm{D}$ reconstruction of true polycrystalline microstructures is now achievable via combination of electron backscatter diffraction (EBSD) mapping and focused ion beam (FIB) serial sectioning techniques [2-8]. Based on these refined frameworks, both property predictions and evolutionary history tracking from meso-scale computational methods such as finite element

Available online at http://link.springer.com/journal/40195

Y.-K. Luo · R.-S. Qin $(\bowtie)$

Department of Materials, Imperial College London, London

SW7 2BP, UK

e-mail: r.qin@imperial.ac.uk

Y.-K. Luo

e-mail: yongkun.luo08@imperial.ac.uk and phase field simulations have become tangible [9-11]. Nevertheless, in contrast to the well-established understandings regarding grain properties, comprehensive quantification of grain boundary (GB) and interface properties, especially their orientation dependence, has yet to be accomplished. The principal hardship of the matter resides in the geometric complexity of GBs. Technical specification of a particular GB requires five macroscopic geometric degrees of freedom (DOF): three DOFs describe the crystallographic orientation relationship (OR) or "misorientation" between the two pertinent lattices, and further two identify the interface plane where the two grains join [12]. Another equivalent commonly seen notation is the axisangle pair presentation. Apart from the macroscopic DOFs, the microscopic relaxations within $\mathrm{GB}$, such as atomic reconstruction and vacancy redistribution, add more dimensions to the already complicated situation. Conventional approaches generally treat the anisotropy of GB properties in a binary manner [13]. For the GBs with relatively small deviation on either misorientation or interface plane from a perfect lattice match, their properties 
are thought to scale metrically according to the corresponding deviations. For the GBs with comparatively high deviations, on the other hand, the properties are considered to be similar to a constant which represents any "random" GB. However, recent work by Olmsted et al. [14] on the GB energy of elementary Ni reported a significant span from 0.6 to $1.4 \mathrm{~J} / \mathrm{m}^{2}$ for the "random" GBs alone, suggesting that the conventional binary treatment on GB properties is too simple and incorrect. An exhaustive 5D study, therefore, is still necessary towards systematic understanding of the matter. Existing models that are able to cope with the five dimensions of GB belong to either atomistic simulations or geometric approaches [12, 14-30]. These two groups possess distinctive strengths and weaknesses. Atomistic simulations, mostly molecular dynamics (MD)-based methods, can calculate the absolute GB energy with consideration of atomistic relaxations, thus are able to provide more precise and accurate results. Nevertheless, the required computational resource is proportional to the simulation cell size, which escalates as the crystallographic complexity of the GB grows. This fact renders it more appropriate for insightful investigation on the properties of a well-defined coherent GB, instead of scanning across the 5D space to assess the full anisotropy of GB properties. A noteworthy contribution of MD-based works can be the construction of grain boundary energy database followed by its usage in phase field modelling (PFM) study for microstructure evolution of $\mathrm{Fe}[11,17]$. However, the database only consider CSL boundaries corresponding to $\sum \leq 33$, whereas other work [14] claims that CSL boundaries with higher $\sum$ values share similar GB energy values. Geometric approaches, on the other hand, attempt to develop rules of general usefulness in the 5D continuum. Current methods that fall in such category include the O-lattice, $\Delta g$ and invariant-line methods. Such approaches quantify either the 2D (GB/interface plane) or 3D (lattice) mismatches of the constituent atoms in the reciprocal space and determine the anisotropic strength accordingly. However, the qualitative nature of most geometric approaches makes them more useful for searching preferable ORs but not a competent quantifying tool.

It would seem that a better approach may be proposed by combining the strengths of both aforementioned categories. Evaluation on existing geometric models reveals that they generally focus on the number of chemical bonds created or broken upon the advent of an interface, but overlook the nature of these interactions. In other words, none of the existing geometric approaches consider the microscopic DOFs that arise from a macroscopically specified GB. This explains why most geometric approaches share the same inability to offer quantifiable results. Brief consideration on the possible relative movements between the relevant lattice planes reveals three microscopic DOFs associated with elastic GB plane relaxations. Note that the microscopic DOFs relating to the "plastic" GB plane relaxations, which consist of symmetry-breaking redistribution of atoms and vacancies along the GB plane, are yet to be discussed.

In this paper, full 5D plots of GB energy are approximated by a newly developed geometric model combined with the Monte Carlo (MC) method. The model allows energy estimation of arbitrarily oriented GBs which cannot be constructed through either MD or experimental methods. Application of the model on elementary facecentred cubic (FCC) metals delivers results that are comparable to those reported in automated EBSD-FIB-based researches $[5,6]$. The article is organized as follows. The theoretical aspects and implementation of the model are covered in Sect. 2. The results are presented in Sect. 3, followed by discussion on top of comparison with recent experimental and MD findings in the literature. Finally, Sect. 4 concludes the present work.

\section{Theory and Implementation}

Thermodynamically, the GB energy discussed here can be defined as the interfacial excess of free energy of the materials. It can be expressed in the Dupré equation [31] as follows:

$\sigma_{12}=\sigma_{1 \mathrm{v}}+\sigma_{2 \mathrm{v}}-W_{\text {sep }}$,

where $\sigma_{12}$ represents the interfacial energy of the two solid species 1 and 2; $\sigma_{1 \mathrm{v}}$ and $\sigma_{2 \mathrm{v}}$ denote the superficial or solid/vapour energy of species 1 and 2 , respectively; $W_{\text {sep }}$ refers to the work of separation for the destruction of such an interface. If Eq. (1) is to be used to assess the 5D GB energy plot, orientation-specified $\sigma_{1 \mathrm{v}}, \sigma_{2 \mathrm{v}}$ and $W_{\text {sep }}$ must be quantified for any arbitrary orientation.

\subsection{Quantification of Orientation-Specified Surface Energy}

Quantification of surface energy for arbitrarily high-indexed orientations via atomistic methods has proven to be difficult, as the surface in question can be tricky to imitate in the simulation cell. On the other hand, if pair potentials are considered instead of $\mathrm{N}$-body potentials, it is possible to assess the unrelaxed specific surface energy for any arbitrary orientations through a geometric approach. In our latest work [32], we proposed a long-range broken-bond model which allows the computation of the surface energy of elementary fcc metal in arbitrary orientations. The model considers up to the fourth nearest neighbouring (fourth $\mathrm{NN}$ ) interactions and produces results which are in 
Table 1 List of parameters used in Eq. (1)

\begin{tabular}{|c|c|c|c|c|c|c|c|c|}
\hline Metal & $a\left(10^{-10} \mathrm{~m}\right)$ & $\phi_{\mathrm{b}^{1}}(e V)$ & $\phi_{\mathrm{b}^{2} / \phi_{\mathrm{b}^{1}}}$ & $\phi_{\mathrm{b}^{3}} / \phi_{\mathrm{b}^{1}}$ & $\phi_{\mathrm{b}^{4}} / \phi_{\mathrm{b}^{1}}$ & $\sum b^{1 d}$ & $\sum b^{2 d}$ & $\sum b^{3 d}$ \\
\hline $\mathrm{Ni}$ & 3.524 & 0.2249 & 0.434 & 0.175 & 0.078 & & & \\
\hline $\mathrm{Cu}$ & 3.615 & 0.1794 & 0.424 & 0.168 & 0.073 & & & $a[800]^{\mathrm{i}}$ \\
\hline $\mathrm{Au}$ & 4.079 & 0.2348 & 0.301 & 0.087 & 0.028 & & & $a[721]^{\mathrm{ii}}$ \\
\hline $\mathrm{Ag}$ & 4.086 & 0.1673 & 0.359 & 0.122 & 0.046 & $a[210]$ & $a[111]$ & $a[640]^{\mathrm{iii}}$ \\
\hline $\mathrm{Pt}$ & 3.924 & 0.3514 & 0.318 & 0.097 & 0.032 & & & $a[552]^{\text {iv }}$ \\
\hline $\mathrm{Pd}$ & 3.891 & 0.2334 & 0.320 & 0.098 & 0.033 & & & $a[633]^{\mathrm{v}}$ \\
\hline $\mathrm{Pb}$ & 4.951 & 0.1213 & 0.323 & 0.100 & 0.034 & & & \\
\hline
\end{tabular}

The $\sum \mathrm{b}^{\text {nd }}$ values are given for the reference region with Miller indices $h \geq k \geq l$ by a geometric approach. Detailed derivation can be found in Ref. [32]

${ }^{\mathrm{i}}$ If $\mathrm{n} \cdot[\overline{1} 21] \leq 0 ;{ }^{\text {ii }}$ if $\mathrm{n} \cdot[\overline{1} 21] \geq 0, \mathrm{n} \cdot[\overline{1} 12] \leq 0$ and $\mathrm{n} \cdot[1 \overline{2} 1] \geq 0$; ${ }^{\text {iii }}$ if $\mathrm{n} \cdot[\overline{1} 12] \leq 0$ and $\mathrm{n} \cdot[1 \overline{2} 1] \leq 0 ;{ }^{\text {iv }}$ if $\mathrm{n} \cdot[\overline{1} 12] \geq 0$ and $\mathrm{n} \cdot[1 \overline{2} 1] \leq 0 ;{ }^{\mathrm{v}}$ if $\mathrm{n} \cdot[\overline{1} 12] \geq 0$ and $\mathrm{n} \cdot[1 \overline{2} 1] \geq 0$

good agreement with experimental findings and other computational methods.

The absolute unrelaxed orientation-specified surface energy can be assessed via the following equation:

$$
\begin{aligned}
\gamma(\hat{\mathrm{n}})= & \frac{\phi_{\mathrm{b}^{1}} \mathrm{n}}{2 \Omega|\hat{\mathrm{n}}|} \\
& \cdot\left(\sum \mathrm{b}^{1 \mathrm{~d}}\left(1+2 \frac{\phi_{\mathrm{b}^{4}}}{\phi_{\mathrm{b}^{1}}}\right)+\sum \mathrm{b}^{2 \mathrm{~d}} \frac{\phi_{\mathrm{b}^{2}}}{\phi_{\mathrm{b}^{1}}}+\sum \mathrm{b}^{3 \mathrm{~d}} \frac{\phi_{\mathrm{b}^{3}}}{\phi_{\mathrm{b}^{1}}}\right)
\end{aligned}
$$

where $\hat{n}$ represents the outward unit normal vector of the surface; $\Omega$ denotes the relevant atomic volume; $\phi_{\mathrm{b}^{1}}$ and the $\phi_{\mathrm{b}^{\mathrm{n}}} / \phi_{\mathrm{b}^{1}}$ terms are bond strength-related coefficients which are determined from the Rose-Vinet universal potential equation [33]; finally, the $\sum b^{\text {nd }}$ terms refer to geometric factors associated with the relevant NN interactions. Table 1 details the input parameters used in the present study for Eq. (2).

A simple EAM-based consideration suggests that the $\mathrm{N}$-body component neglected by the above approach is about $3 \% E_{\mathrm{c}}$ depending on the choice of the empirical model.

\subsection{Quantification of Orientation-Specified Work of Separation}

In a simple assumption, the work of separation can be treated as the work required to overcome the resulting chemical binding energy across the relevant interface. Such a concept is comparable to the "cleavage energy" term proposed by Wolf [24]. In a geometric, pair potential-based approach, where the bond strengths are solely dependent on interatomic distances, the total chemical binding energy can be evaluated simply by sampling all interactions between the atoms within the effective potential radius of a reference point which resides on the interface plane. If the atomistic relaxations are neglected, then there is no orderbreaking redistribution of atoms or vacancies along the interface. Such simplification circumvents the usage of time-step methods and allows quick scanning over vast amounts of orientations. The relative impacts of the elastic and plastic GB plane relaxations on the GB energy can be peeked from the works by Wolf [24], in which the correlations between structure, energy and ideal cleavage for symmetrical GBs in fcc metals were studied. It is proposed that the elastic relaxations play a critical role, whereas the plastic relaxations, which scale with the GB complexity, have a relatively small effect on GBs with comparatively low energy. We aim for a model which is able to cope with elastic GB plane relaxations when the "plastic" GB plane relaxations are neglected.

It should be reminded that the binding energy obtained for a macroscopically specified point is not unique but greatly depends on the initial configuration of the actual GB. As such, the orientation, according to the quantification of GB properties, a seemingly 5D problem, is indeed an $8 \mathrm{D}$ problem of finding the minimum in the microscopic $>3 \mathrm{D}$ subspace for each definable point in the 5D macroscopic space. The goal here, therefore, is not to compute a single absolute energy value, but to search for the maximum in the microscopic domain for every point defined in the macroscopic space. Although exhaustion of all nonequivalent starting configurations is theoretically possible, the actual number of these configurations varies significantly from one GB to another. Olmsted and colleagues reported numbers from 500 to 50,000 depending on the GB complexity [14]. Additionally, given the black-box nature of the microscopic domain, finding the global maximum of the work of separation, or equivalently the global minimum of interface energy, cannot be guaranteed. It should also be noted that, in our approach, some of the essentially equivalent configurations may yield different energy values 
as atomistic relaxations are overlooked. This fact will boost the already considerable number of non-equivalent starting configurations to an even higher scale.

Based on the above concerns, instead of carefully sorting out the non-equivalent initial configurations for each GB, we decide to adopt a Monte Carlo (MC)-based method for the maximum search. According to the work by Olmsted et al., though varies largely in numbers, the majority (more than 50\%) of non-equivalent starting configurations returned the same energy value, which essentially is, or at least very close to, the found minimum. Moreover, there are quite a few (up to $10 \%$ ) configurations demonstrating much higher energy values than that of the majority, but the gap between the found minimum and the most populated values is small $(<5 \%)$. This suggests that the difference between the most populated energy values and the found minimum may be of second-order importance. If we assume the configurations are evenly distributed in space and consider a 99\% confidence interval, performing 1000 random searches in the corresponding domain will cover $99.5 \%$ of all candidate configurations. We believe such a method is sufficiently competent for the present goal.

\subsection{Implementation}

Consider a randomly sampled GB segment in practice. Among its five macroscopic DOFs, the three regarding misorientations are generally unchanged as the parent grains cannot rotate freely, whereas the other two representing GB plane orientation are probably continuous for any non-straight GB. With this fact in mind, we decide to format our output as separate projections sorted against their corresponding OR matrices. If two abutting lattices are denoted as $X$ and $Y$, the corresponding OR matrix, namely the coordinate transformation matrix which transfers the vector presentation from the coordinate system of $X$ to that of $Y$, can be referred to as $(Y J X)$ if $X$ is treated as the reference lattice. Detailed approaches regarding transforming axis-angle pair presentations, e.g. $\langle 111\rangle 60^{\circ}$ to their equivalent $(Y J X)$ can be found in the textbook by Bhadeshia [34].

The present work on elementary fcc metals began with the construction of two identical supercells of $X$, one of which is then transformed into a supercell of $Y$. After that, a specific GB plane orientation is chosen, and the anisotropic surface energy is calculated through Eq. (2). A reference point is then randomly generated within the primitive cell located in the middle of each of the two supercells. In the next step, the two supercells are both halved by a plane of chosen orientation across their corresponding reference points, before the halves are brought together with the reference points coinciding each other. A cylindrical region
Table 2 List of the studied coordinate transformation matrices (YJX) together with their equivalent CSL and axis-angle pair notations

\begin{tabular}{|c|c|c|c|c|}
\hline \multirow{2}{*}{$\begin{array}{l}\text { Coincidence site } \\
\text { lattice (CSL) } \\
\text { notation }\end{array}$} & \multirow{2}{*}{$\begin{array}{l}\begin{array}{l}\text { Axis-angle } \\
\text { pair } \\
\text { notation }\end{array} \\
\langle 111\rangle 60^{\circ}\end{array}$} & \multicolumn{3}{|c|}{$\begin{array}{l}\text { Coordinate transformation } \\
\text { matrix }(Y J X)\end{array}$} \\
\hline & & $\left(\begin{array}{r}0.667 \\
-0.333 \\
0.667\end{array}\right.$ & $\begin{array}{r}0.667 \\
0.667 \\
-0.333\end{array}$ & $\left.\begin{array}{r}-0.333 \\
0.667 \\
0.667\end{array}\right)$ \\
\hline$\sum 5$ & $\langle 100\rangle 36.86^{\circ}$ & $\left(\begin{array}{rr}1 & 0 \\
0 & 0.8 \\
0 & -0.6\end{array}\right.$ & $\begin{array}{cc} & 0 \\
8 & 0.6 \\
6 & 0.8\end{array}$ & \\
\hline$\sum 7$ & $\langle 111\rangle 38.21^{\circ}$ & $\left(\begin{array}{r}0.857 \\
-0.286 \\
0.429\end{array}\right.$ & $\begin{array}{r}0.429 \\
0.857 \\
-0.286\end{array}$ & $\left.\begin{array}{r}-0.286 \\
0.429 \\
0.857\end{array}\right)$ \\
\hline$\sum 9$ & $\langle 110\rangle 38.94^{\circ}$ & $\left(\begin{array}{l}0.889 \\
0.111 \\
0.444\end{array}\right.$ & $\begin{array}{r}0.111 \\
0.889 \\
-0.444\end{array}$ & $\left.\begin{array}{r}-0.444 \\
0.444 \\
0.778\end{array}\right)$ \\
\hline$\sum 11$ & $\langle 110\rangle 50.47^{\circ}$ & $\left(\begin{array}{l}0.818 \\
0.182 \\
0.545\end{array}\right.$ & $\begin{array}{r}0.182 \\
0.818 \\
-0.545\end{array}$ & $\left.\begin{array}{r}-0.545 \\
0.545 \\
0.636\end{array}\right)$ \\
\hline$\sum 17 b$ & $\langle 221\rangle 61.9^{\circ}$ & $\left(\begin{array}{c}0.894 \\
0.029 \\
1\end{array}\right.$ & $\begin{array}{r}0.818 \\
0.894 \\
-0.577\end{array}$ & $\left.\begin{array}{c}-0.577 \\
1 \\
0.577\end{array}\right)$ \\
\hline$\sum 31 \mathrm{a}$ & $\langle 111\rangle 17.9^{\circ}$ & $\left(\begin{array}{r}0.968 \\
-0.161 \\
0.194\end{array}\right.$ & $\begin{array}{r}0.194 \\
0.968 \\
-0.161\end{array}$ & $\left.\begin{array}{r}-0.161 \\
0.194 \\
0.968\end{array}\right)$ \\
\hline
\end{tabular}

Note that, for any specific transformation regarding cubic systems, there exist multiple $(Y J X)$ s which lead to the same end state

centred at the combined reference point with its basal planes parallel to the GB plane is subsequently picked for the calculation of the binding energy. For every overlapping atom pair, we insert an atom in their mean position and vary the cut-off distance from 0.5 to 0.8 equilibrium first NN distance. The above process is treated as the construction of a single starting configuration and is repeated for 1000 more times during which the maximum binding energy is recorded. The approximate absolute value for this specific GB plane orientation is then computed via Eq. (1), before the programme scans through the rest of the GB plane orientations for the OR of interest.

\section{Results and Discussion}

The northern stereographic projections of pure twist $\sum 3$, $\sum 5, \sum 7, \sum 9, \sum 11, \sum 17 \mathrm{~b}$ and $\sum 31 \mathrm{a}$ ORs are constructed. The detailed $(Y J X)$ matrices and their equivalent axis-angle pair presentation are listed in Table 2. Each of the projections consists of 8320 data points among which the colormap was interpolated. The figures are plotted as scattered points. The figures are based on results of elementary $\mathrm{Ni}$, regarding which readily comparable results 
exist in the literature. Projections of other FCC metals exhibit similar topographic patterns.

As a general remark, the figures demonstrate complex yet distinct patterns which vary greatly from one OR to another. The results of the $\sum 3$ OR, which is given in Fig. 1a, unsurprisingly shows a global minimum on the (111) pole, where the coherent twinning resides. It also demonstrates that two strong threefold symmetries radiated from the (111) pole towards the $\{100\}$ and $\{110\}$, respectively. A number of smaller and shallower cusps can be seen but indicate no apparent symmetry. Figure $1 \mathrm{~b}$ depicts the pole diagram of the $\sum 5 \mathrm{OR}$, where full cubic symmetry seems to have been restored. A number of equally weighted local minimums can be seen located on the $\{111\},\{100\}$ and $\{110\}$ poles. Figure 1c, showing the projection of the $\sum 7 \mathrm{OR}$, also strongly favours the (111) orientation. Unlike Fig. 1a, however, the small cusps now sit on the $\langle 110\rangle$ zone circles and follow no simple symmetries. The pattern presented in the $\sum 9$ OR plot, given in Fig. 1d, differs from the previous three by showing a distinctive "groove" along the [110] zone circle. Figure 2a, which carries the projection of $\sum 11$, somewhat resembles Fig. 1d, but possesses fewer yet deeper cusps on the (001) and $(1 \overline{1} 1)$ poles. The anisotropy of the $\sum 17 \mathrm{~b}$ and the $\sum 31$ a ORs, which possess relatively high $\sum$ values, is mapped in Fig. 2b, c, respectively. Both of these figures
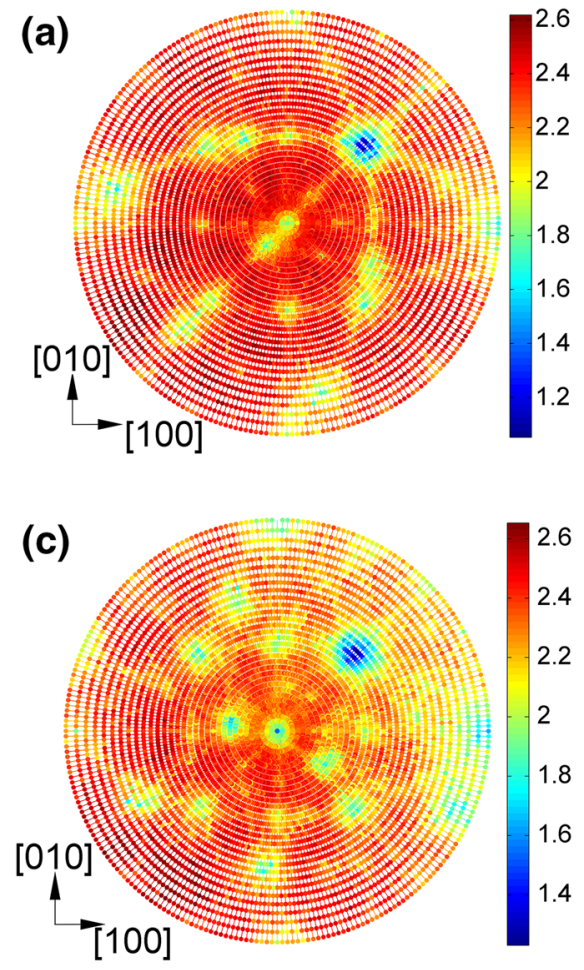

show little similarity to the previously discussed results. Figure $2 b$ shows a spread of cusps along the [100], [011] and $[0 \overline{1} 1]$ zone circles, whereas Fig. 2c demonstrates two crossing hourglass-shaped regions.

A prominent impact of GB plane inclination on GB energy is observed in our finding. This means that the anisotropy of GB properties cannot be meaningfully depicted by disorientation angle alone. The studied ORs show similar spreads of calculated energy values, with an exception of $\sum 17 \mathrm{~b}$ OR, which exhibits systematically higher energy values than the rest. This suggests that the spread of GB energy for a given OR may be largely controlled by its misorientation axes. The $\sum$ number, which represents the coincident site density of the joined lattices in the reciprocal space, was found to have no conclusive influence on the number, strength or location of favoured poles. Twist boundaries with $\langle 111\rangle$ rotation axis always exhibit cusps in the vicinity of (111) pole. These findings are in agreement with several recent remarks [5, 14, 18, 29, 30].

Our results demonstrate a striking similarity to experimentally observed grain boundary energy distribution (GBED) figures [5, 6]. In particular, the topographies of highly populated $\sum 3$ and $\sum 9$ ORs are faithfully resembled. For the scarcely sampled $\sum 5$ and $\sum 7$ ORs, somewhat larger discrepancies are seen. It should be reminded that, in practice, the GBs are sampled according to
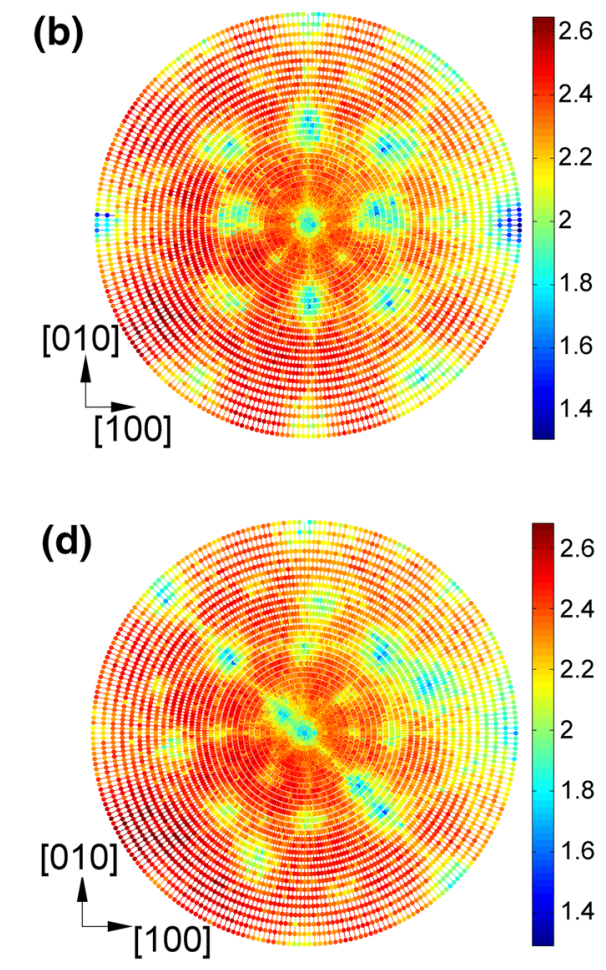

Fig. 1 The corresponding northern stereo projections of Ni grain boundaries with a $\langle 111\rangle 60^{\circ} \sum 3 \mathrm{OR}, \mathbf{b}\langle 100\rangle 36.86^{\circ} \sum 5 \mathrm{OR}$, $\mathbf{c} \sum 7 \mathrm{OR}$ and $\mathbf{d}$ $\langle 110\rangle 38.94^{\circ} \sum 9$ OR (the plots are viewed along the [001] axis; energy $\left(\mathrm{J} / \mathrm{m}^{2}\right)$ is dyed from blue to red in ascending order) 

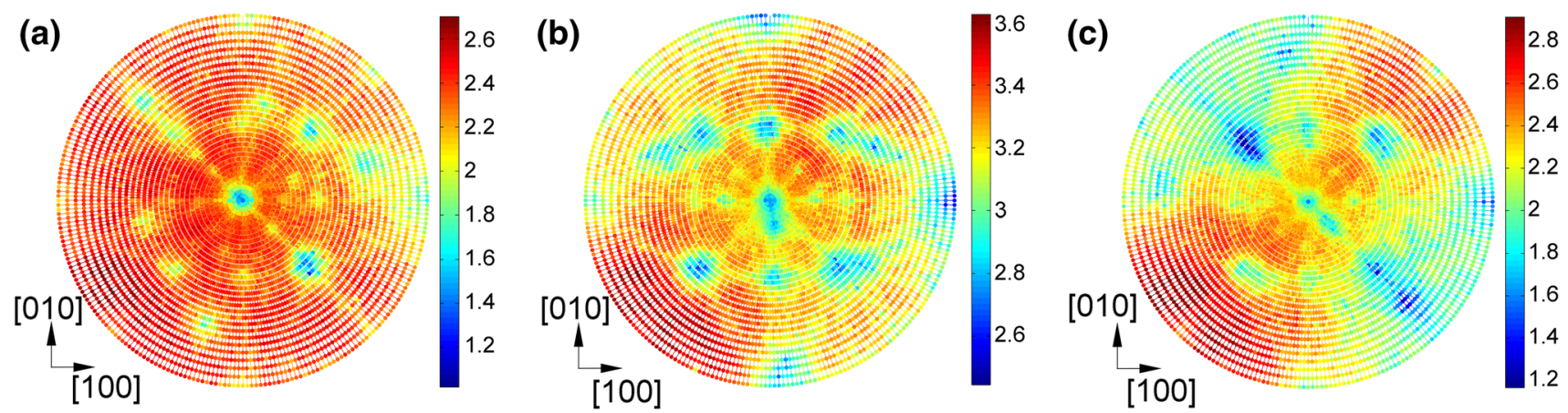

Fig. 2 The corresponding northern stereo projections of Ni grain boundaries with a $\langle 110\rangle 50.47^{\circ} \sum 11$ OR, b $\langle 221\rangle 61.9^{\circ} \sum 17 \mathrm{~b}$ OR and $\mathbf{c}$ $\langle 111\rangle 17.9^{\circ} \sum 31 \mathrm{a}$ OR (the plots are viewed along the [001] axis; energy $\left(\mathrm{J} / \mathrm{m}^{2}\right)$ is dyed from blue to red in ascending order)

Brandon's criterion under room temperature, with their orientations somehow artificially smoothed. As such, the high portion of our $0 \mathrm{~K}$ anisotropy features retained in GBED figures is unexpected. In this sense, the true anisotropy of GB energy may be more significant than what was previously believed. On the other hand, despite possessing similar spreads of GB energy, the $\sum 9$ OR GBs are observed in a distinctively higher population than those of $\sum 5$ and $\sum 7$ ORs $[5,35]$. This also contradicts with recent MD findings which suggest that $\sum 5$ and $\sum 9$ ORs share similar spread of absolute GB energy values, both higher than that of $\sum 7$ [14]. Based on these informations, one may think that the relative abundance of a particular GB may not be solely determined by its GB energy values. Although seemingly surprising, the above statement can be easily justified by the texture dependence of alloys on their thermomechanical processing history. Considering the observed case of $\sum 9$ OR GBs, it may be hypothesized that the practically favoured ORs do not necessarily possess absolutely low GB energy values, but instead show grooves along which dislocation movements are favoured. The above hypothesis is consistent with the fact that curved GBs are commonly observed. Another point to note is that the "real" GBs measured in reality might not reside on the optimum states, as doing so may render contiguous GBs more energetically unfavourable, thus raise the energy of the whole system. As such, how to estimate the "real" GB properties, which are likely to be associated with metastable states, is still a big challenge. These thoughts may be better answered by expanding current research to other lattice systems.

It is interesting to note that our calculated GB energy values are higher than molecular dynamic (MD)-based values by a consistent magnitude of $1 \mathrm{~J} / \mathrm{m}^{2}$ for $\mathrm{Ni}$ [14]. In this work, the minimums found for the $\sum 5, \sum 7$ and $\sum 9$ OR are $1.31,1.23$ and $1.28 \mathrm{~J} / \mathrm{m}^{2}$, respectively, which are not much higher than the $1.06 \mathrm{~J} / \mathrm{m}^{2}$ value found for the $\sum 3$ coherent twinning. The corresponding values given by Olmsted et al. were roughly $0.9,0.5,0.9$ and $0.06 \mathrm{~J} / \mathrm{m}^{2}$, respectively. The maximum energy value found in this work for each OR is consistently around $2.65 \mathrm{~J} / \mathrm{m}^{2}$ except for $\sum 17 \mathrm{~b}$, which demonstrates a spread with roughly $1 \mathrm{~J} / \mathrm{m}^{2}$ higher. Olmsted et al. [14] reported values of between 1.4 and $1.6 \mathrm{~J} / \mathrm{m}^{2}$, which are $1 \mathrm{~J} / \mathrm{m}^{2}$ lower. Consider the present work is sampling much more GB than the MD studies, it is reasonable for our results to demonstrate a relatively wider spread of GB energy. We believe that this consistent difference is mainly caused by our overlooking of plastic GB plane relaxations and the multi-body influences. The fact that our minimum search method is somewhat coarse compared to those employed in MD simulations might also be part of the cause, and increasing MC iteration number will definitely help. However, consider the fact that a true minimum must be a minimum in terms of both elastic and plastic GB plane relaxations, and any cusps reported in the current work must be retained in the true 5D topography, though their absolute depths might be wrongly estimated. In other words, it is difficult to imagine that the full characteristics of a freshly created GB will be completely destroyed in the subsequent redistribution of atomic species. A good example of this can be the lowenergy $\sum 11\langle 110\rangle 50.47^{\circ}(1 \overline{1} 3) /(\overline{1} 13)$ GB which is discovered by Hasson et al. [36]. Our results suggest a value of $1.8 \mathrm{~J} / \mathrm{m}^{2}$ with a shallow yet distinct cusp of this particular GB in Fig. 2a, whereas Olmsted et al. [14] reported a value of $0.4 \mathrm{~J} / \mathrm{m}^{2}$. Note that this particular GB is not one of the global minimums of $\sum 11 \mathrm{OR}$, which are located on the $(001)$ and $(1 \overline{1} 1)$ poles. One noteworthy explanation of this matter can be that the $(1 \overline{1} 3) /(\overline{1} 13)$ GB is associated with much higher plastic relaxation energy when compared with the two "fake" poles and thus is indeed the global minimum. Or, alternatively, as the two deepest cusps are located in the vicinity of $(1 \overline{1} 1) /(001)$ GB in the 5D continuum, they may be the true cusps for this OR, but their population is somehow diluted by the Brandon's criteria employed in the characterization process. 
It should be highlighted that our results provide GB energy values for any arbitrary orientations, which are very useful input parameters in meso-scale simulation tools [37]. Some may argue that the values regarding irrational orientations have no physical meaning, as these GBs do not exist naturally. Surely, in reality, two adjacent, arbitrarily complex GBs may well possess identical structure when fully relaxed. However, in practice, the relative "unstabilities" of these GB must be mathematically explained in the simulation tools to offer convincing results. In these cases, we are more interested in how quick and how much the GB energy changes across the 5D continuum. The current model may not be best suited for insightful researches regarding GBs with well-recognized characteristics, but is useful for systems where a general understanding has not been established. The method also works as a simple approach to provide ready-to-use input GB energy data for meso-scale simulations, one that can be easily integrated into other frameworks. Here, we have kept the model as cleans as possible in the hope that potential users may parametrize it in the ways they see fit. Finally, as a completely general method for the calculation of GB energy, this model has yet to be tested for various types of GBs involving metal/metal, ionic/ionic and metal/ ionic interfaces to determine its range of validity and predictive power. It will be of great interest to expand the current work into the above-mentioned fields.

\section{Conclusions}

Quantification of GB energy across the full 5D macroscopic continuum has been achieved by a newly proposed geometric method. Northern stereo projections of typical $\sum 3, \sum 5, \sum 7, \sum 9, \sum 11, \sum 17 \mathrm{~b}$ and $\sum 31 \mathrm{a}$ ORs are constructed for elementary Ni. The topographies are in general complex with no apparent trends to be concluded. Both misorientation and GB plane inclination are found to have a prominent impact on the energy values. The number, depth and position of cusps show no conclusive relationship against the $\sum$ values, but are found to be dependent on the misorientation rotation axes. GBs located in the vicinity of the $\{111\}$ poles are generally favoured in terms of energy. The plots for $\sum 3, \sum 5, \sum 7$ and $\sum 9$ ORs share the main features of experimentally determined GBED figures. The calculated values of absolute GB energies demonstrated a similar window of spread as compared to MD findings, but are consistently higher in magnitude by $1 \mathrm{~J} / \mathrm{m}^{2}$ for Ni. Parametric fittings based on the current framework to MD results may be powerful supplementaries to existing meso-scale simulations on polycrystalline microstructures.

Acknowledgments The authors are grateful for the financial support from TATA Steel and the Royal Academy of Engineering.

\section{References}

[1] S.E. Offerman, Science 305, 190 (2004)

[2] D.M. Saylor, A. Morawiec, G.S. Rohrer, Acta Mater. 51, 3663 (2003)

[3] J. Konrad, S. Zaefferer, D. Raabe, Acta Mater. 54, 1369 (2006)

[4] M. Groeber, B. Haley, M. Uchic, D. Dimiduk, S. Ghosh, Mater. Charact. 57, 259 (2006)

[5] V. Randle, R. Jones, Mater. Sci. Eng. A 524, 134 (2009)

[6] J. Li, S.J. Dillon, G.S. Rohrer, Acta Mater. 57, 4304 (2009)

[7] H. Beladi, G.S. Rohrer, A.D. Rollett, V. Tari, P.D. Hodgson, Acta Mater. 63, 86 (2014)

[8] H. Beladi, G.S. Rohrer, Acta Mater. 61, 1404 (2013)

[9] A.J. Wilkinson, T.B. Britton, Mater. Today 15, 366 (2012)

[10] M. Kartal, F. Dunne, A.J. Wilkinson, Acta Mater. 60, 5300 (2012)

[11] H.K. Kim, S.G. Kim, W. Dong, I. Steinbach, B.J. Lee, Model. Simul. Mater. Sci. Eng. 22, 034004 (2014)

[12] A.P. Sutton, R.W. Balluffi, Interfaces in Crystalline Materials (Oxford University Press, Hoboken, 1995)

[13] Q. Jiang, H. Lu, Surf. Sci. Rep. 63, 427 (2008)

[14] D.L. Olmsted, S.M. Foiles, E.A. Holm, Acta Mater. 57, 3694 (2009)

[15] A.P. Sutton, R.W. Balluffi, Acta Metall. 35, 2177 (1987)

[16] V.V. Bulatov, B.W. Reed, M. Kumar, Acta Mater. 65, 161 (2014)

[17] H.K. Kim, W.S. Ko, H.H. Lee, S.G. Kim, B.J. Lee, Scr. Mater. 64, $1152(2011)$

[18] E.A. Holm, D.L. Olmsted, S.M. Foiles, Scr. Mater. 63, 905 (2010)

[19] A.R.S. Gautam, J.M. Howe, Philos. Mag. 91, 3203 (2011)

[20] F. Dai, W. Zhang, Model. Simul. Mater. Sci. Eng. 21, 075002 (2013)

[21] Y. Shibuta, S. Takamoto, T. Suzuki, ISIJ Int. 48, 1582 (2008)

[22] D. Wolf, J. Appl. Phys. 68, 3221 (1990)

[23] D. Wolf, Acta Metall. Mater. 38, 781 (1990)

[24] D. Wolf, J. Mater. Res. 5, 1708 (1990)

[25] D. Wolf, Acta Metall. Mater. 38, 791 (1990)

[26] D. Wolf, Acta Metall. 37, 2823 (1989)

[27] D. Wolf, Acta Metall. 37, 1983 (1989)

[28] C. Luo, G. Weatherly, Acta Metall. 35, 1963 (1987)

[29] M.A. Tschopp, D.L. McDowell, Philos. Mag. 87, 3147 (2007)

[30] M.A. Tschopp, D.L. McDowell, Philos. Mag. 87, 3871 (2007)

[31] M. Finnis, J. Phys.: Condens. Mater. 8, 5811 (1996)

[32] Y. Luo, R. Qin, Surf. Sci. 624, 103 (2014)

[33] J. Rose, J. Smith, F. Guinea, J. Ferrante, Phys. Rev. B 29, 2963 (1984)

[34] H.K.D.H. Bhadeshia, Worked Examples in the Geometry of Crystals, 2nd edn. (The Institute of Materials, London, 2001)

[35] V. Randle, G.S. Rohrer, H. Miller, M. Coleman, G. Owen, Acta Mater. 56, 2363 (2008)

[36] G. Hasson, J.Y. Boos, I. Herbeuval, M. Biscondi, C. Goux, Surf. Sci. 31, 115 (1972)

[37] I. Steinbach, Model. Simul. Mater. Sci. Eng. 17, 073001 (2009) 